\title{
IDIOPATHIC SCLEROMALACIA: CASE REPORT.
}

Pablo Duarte de Lima (UNIPÊ, João Pessoa, PB, Brasil), Teresa Patrícia Acebey Crespo (UNIPÊ, João Pessoa, PB, Brasil), Monique Martins Alves (UNIPÊ, João Pessoa, PB, Brasil), Marina Brasileiro Leitão (UNIPÊ, João Pessoa, PB, Brasil), Thaynara Sarmento Oliveira (UNIPÊ, João Pessoa , PB, Brasil)

\section{BACKGROUND}

Scleromalacia perforating is a disease characterized by progressive thinning of the sclera and that, in isolated cases, may affect women with rheumatoid arthritis, besides being potentially related to infections and other systemic pathologies or Even being idiopathic .

\section{CASE REPORT}

M. P, female, 73 years old, reported that about 40 years ago began to experience retro-ocular pain, pruritus, photophobia, Conjunctival hyperemia, periorbital edema and tearing in both eyes. It was accompanied by several specialists without an etiological diagnosis defined for long periods. At the time, she was treated with symptomatic and topical corticoids, evolving with pain improvement. In 1991 it evolves with pain of strong intensity $(10 / 10)$ in the left eye, increased ocular pressure of this same eye, besides the change in the coloration of the left sclera, which became bluish. After adequate investigation, it was suggested as a secondary to the possible infection by the human T-lymphotropic virus type 1 (HTLV1-1). Hypothesis discarded after the collection of serial serologies for infection by Citamegalovirus, HIV, toxoplasmosis, herpes, hepatitis B and C, besides syphilis. In 2019, the patient is referred to the rheumatologist with the suspicion of scleroderma associated with rheumatoid arthritis and/or other autoimmune causes. Laboratory results showed: non-detectable HLA-B27, non-reagent FAN, Anti-CCP $12 \mathrm{U}$ and Negative rheumatoid factor (RF), C-reactive protein $=4.3 \mathrm{mg} / \mathrm{L}$, creatinine $=1.3 \mathrm{~m} / \mathrm{dL}$, urea $=74$ $\mathrm{mg} / \mathrm{dl}, \mathrm{AST}=29 \mathrm{U} / \mathrm{L}, \mathrm{ALT}=18$, alkaline phosphatase $=309 \mathrm{U} / \mathrm{L}$ and gamma $\mathrm{GT}=77 \mathrm{U} / \mathrm{L}$. Current ophthalmologic evaluation with visual acuity of 20/25 in the right eye, with the best correction, and visual acuity lower than 20/40 in the left eye, preservation of light perception, decreased left visual field, direct and consensual photomotor reflexes Abolished in both eyes, as well as the reflection of pupillary accommodation, in addition to dyscoic pupils. It currently uses dorzolamide Hydrochloride in association with Timolol Maleate.

\section{CONCLUSION}

Perforating scleromalacia may be associated with possible systemic pathologies such as infectious diseases, neoplasms, degenerative conditions, besides that it may be an extra-articular manifestation of some diseases Rheumatologic and/or autoimmune diseases, such as Sjogren's syndrome, spondyloarthritis and, mainly, rheumatoid arthritis. After excluding the possible differential diagnoses, it was concluded that it was an idiopathic perforating scleromalacia. 